\title{
Ultrafast Non-inverting Wavelength Conversion by Cross-Absorption Modulation in Silicon Wire Waveguides
}

\author{
L.R. Nunes ${ }^{1}$, T.K. Liang ${ }^{1}$, H.K. Tsang ${ }^{2}$, M. Tsuchiya ${ }^{1}$, D. Van Thourhout ${ }^{3}$, P. Dumon ${ }^{3}$, and R. Baets ${ }^{3}$ \\ 1: NICT - National Institute of Information and Communications Technology, Tokyo, Japan. \\ 2: The Chinese University of Hong Kong, N.T., Hong Kong \\ 3: Ghent University - IMEC, Department of Information Technology (INTEC), Gent, Belgium \\ e-mail: nunes@nict.go.jp
}

\begin{abstract}
We demonstrate all-optical wavelength conversion by means of cross absorption effect induced by Two Photon Absorption (TPA) in submicron sized Si wire waveguides. Optical pulses of $1.6 \mathrm{ps}$ at $1 \mathrm{GHz}$ repetition rate were successfully converted from $1550 \mathrm{~nm}$ to $1532 \mathrm{~nm}$. We discuss the performance at higher speed. Our results showed that silicon waveguides have potential applications in high speed photonic signal processing.
\end{abstract}

\section{Introduction}

The high index difference $(\Delta>41 \%)$ in silicon-on-insulator (SOI) platforms allows the realization of submicron size single mode planar waveguides [1]. Due to the high confinement of optical fields in such waveguides, ultra-high optical intensity can be easily achieved with input optical powers typically used in telecommunications.

The high optical intensities in long interaction lengths may lead to the manifestation of different nonlinear optical effects. Recently, different nonlinear applications in silicon waveguides have been reported in the literature, e.g. Stimulated Raman Scattering amplificationlasing [2], and wavelength conversion by cross phase modulation [3], among others.

Most of the reported switching devices in silicon are based on plasma dispersion effect. In such devices, excess free carriers are introduced inside the waveguide either by external current injection [4] or optical excitation [5] to introduce the required absorption or phase shift. In such cases, the speed is limited by the effective carrier lifetime [6].

As TPA is an ultrafast nonlinear process in semiconductors waveguides [7], there is high potential for exploiting it for wideband high speed all-optical devices.

In this paper we report the use of crossabsorption induced by TPA for wavelength conversion. Optical pulses of $1.6 \mathrm{ps}$ with a repetition rate of $1 \mathrm{GHz}$ were converted from the original wavelength of $1550 \mathrm{~nm}$ to $1532 \mathrm{~nm}$.

\section{Experiments}

Figure 1 shows the dimensions of the silicon wire waveguide used in the experiment. The fabrication and characterization of the waveguide was described in [8]. The waveguide is $10 \mathrm{~mm}$ in length, formed in spiral to fit in a very small footprint area. The waveguide core is formed by a silicon strip measuring $480 \mathrm{~nm}$ in width and $220 \mathrm{~nm}$ in height. The buried oxide (BOX) layer is $1 \mu \mathrm{m}$.

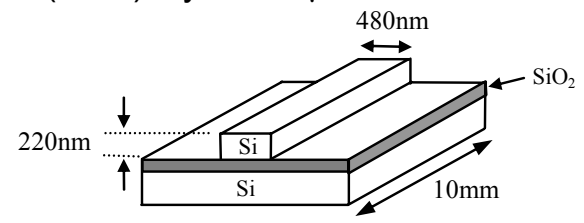

Fig. 1 Silicon wire waveguide dimensions

Figure 2 shows the experimental setup. A mode-locked fiber ring laser was used to generate $1.6 \mathrm{ps}$ pulses with $1 \mathrm{GHz}$ repetition rate at $1550 \mathrm{~nm}$. The pump pulses were boosted up to high power by an erbium-doped fiber amplifier (EDFA). The continuous-wave (CW) signal was generated from a tunable laser operating at $1532 \mathrm{~nm}$. A 70:30 optical coupler combined the pump and CW light respectively. Subsequently, the combined signals are coupled into the wire waveguide by using a grating-based fiber-coupling scheme, whose coupling efficiency is about $20 \%$ [8]. Another EDFA was placed after the waveguide to compensate the insertion loss. We used then an optical bandpass filter (BPF) to filter out the pump pulses at the waveguide output. 


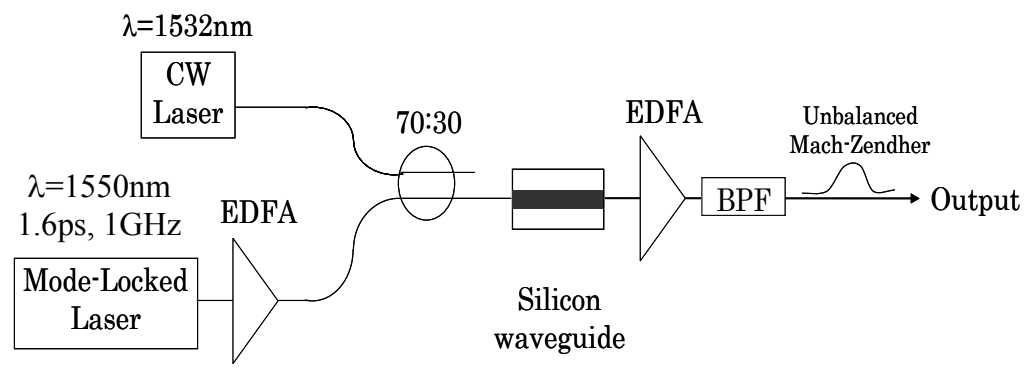

Fig. 2 Experimental Setup

The unbalanced Mach-Zehnder interferometer after the BPF ensured that the cross-modulation was non-inverting at the output. Finally, the wavelength converted output was measured via a 10 $\mathrm{GHz}$ bandwidth photodiode.

\section{Results and Discussion}

The optical spectrum of combined signals (CW and pulse) at the waveguide output is shown in Fig. 3. The spectrum of the converted signal $(\lambda=1532 \mathrm{~nm})$ after the pump pulse suppression is shown in Fig. 4. To remove the residual pump pulse at the output, a high extinction ratio optical bandpass filter of $1 \mathrm{~nm}$ ( $3 \mathrm{~dB}$ bandwidth) is used. As shown in Fig. 4, the ratio of CW signal and pump pulses was greater than $60 \mathrm{~dB}$.

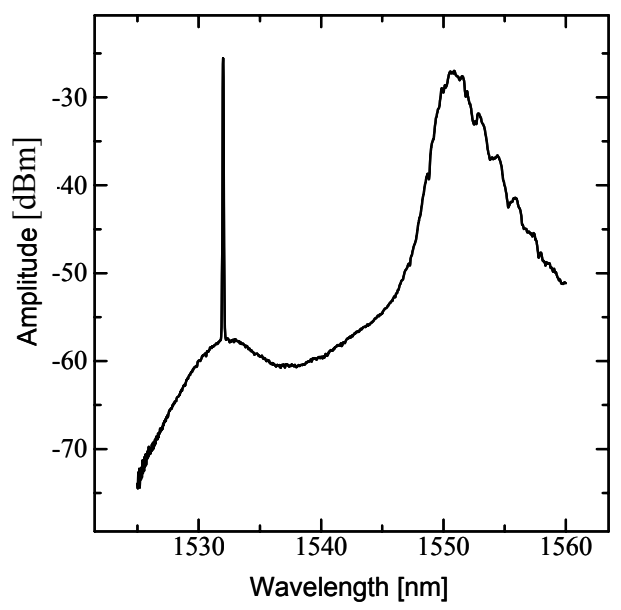

Fig. 3 Optical spectrum after waveguide

With both strong pump pulses and the weak CW signal propagating inside silicon waveguide, one photon from the pump and another photon from the signal will be simultaneously absorbed within the pulse duration, as the sum of their photon energies is greater than the bandgap of silicon. This nondegenerate TPA process
[9], is usually observed only at high optical pump intensities. However, in submicron silicon wire waveguides, as the effective area of optical mode field profile is in the order of $0.1 \mu \mathrm{m}^{2}$, ultrahigh optical intensity can easily be achieved at moderate pump power levels.

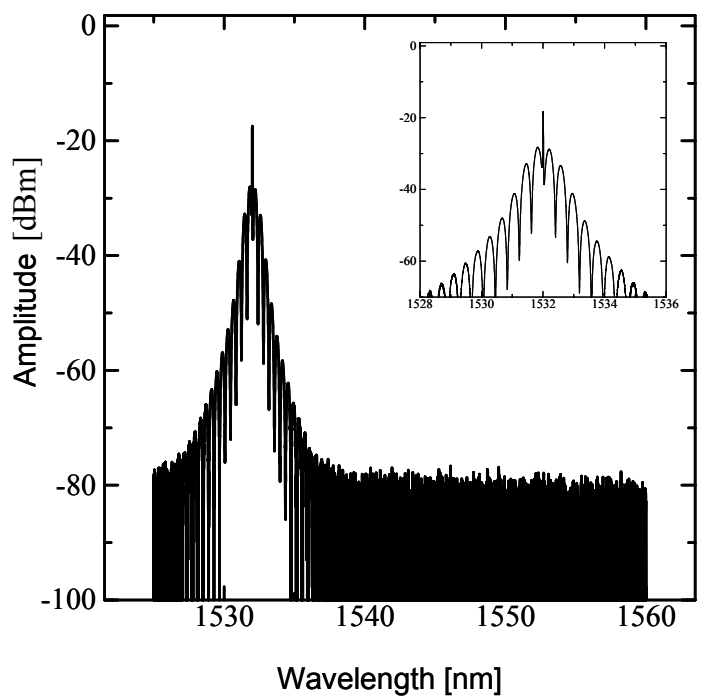

Fig. 4 Output optical spectrum (after the Mach-Zendher interferometer)

Figure 5 shows the time domain of the wavelength converted signal after the band pass filter, observed in an oscilloscope with a $10 \mathrm{GHz}$ bandwidth photodiode. The signal is converted in the form of dark pulses. The original $1.6 \mathrm{ps}$ pulsewidth was broadened to around 60 ps due to the slow response of the used photodiode. The cross-modulated signal consists of a fast transient process and a slow recovery component. The fast component has almost the same pulsewidth as the original pump pulse. The pumpinduced fast absorption is attributed to the ultrafast TPA process. The slow recovery component was due to free carrier absorption because TPA will generate 
excess free carriers inside the waveguide. Since we used ultra-short pump pulses (1.6 ps), only a small amount of free carriers were generated. Therefore, the effect of long lived free-carrier absorption is nearly negligible.

Due to the slow photodiode response, the actual modulation depth might be much better than the observed one.

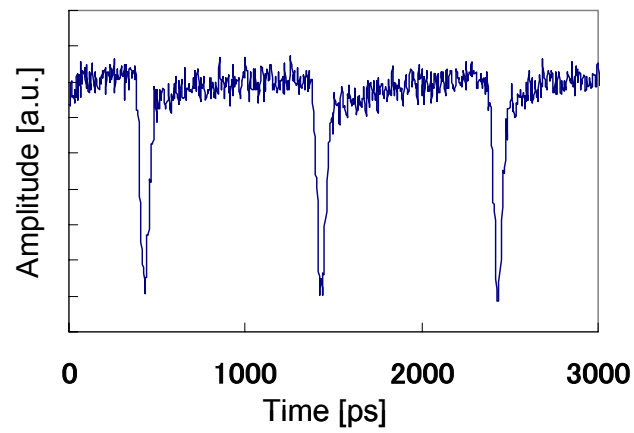

Fig. 5 Modulated CW signal at 1532 nm- Dark pulses. (before the Mach-Zendher interferometer)

The unbalanced Mach-Zehnder shown in the experimental setup is used to convert the dark-pulses into light-pulses. Fig. 6 shows the light-pulses at the circuit output.

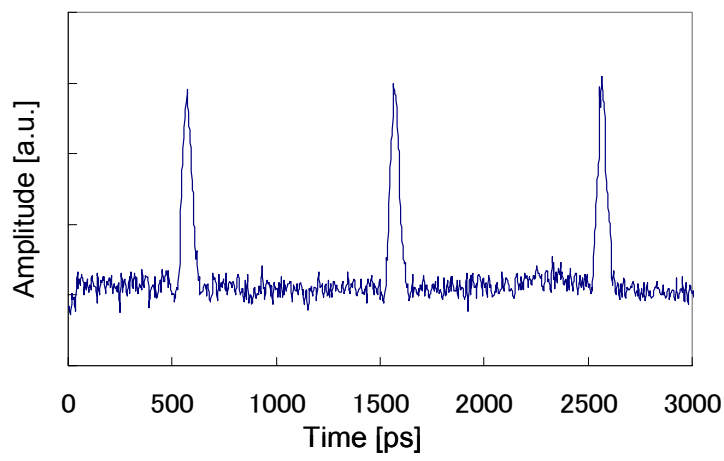

Fig. 6 Modulated cw signal at $1532 \mathrm{~nm}$ - Light pulses. (after the Mach-Zendher interferometer)

Our results suggested that wavelength conversion at higher repetition rate is possible if the pump pulses are short enough to generate only a small amount of free carriers.

The wavelength difference between pump and signal in our experiment was only 18 $\mathrm{nm}$. However, as the conversion mechanism is based on TPA process, the signal wavelength can be anywhere between $1200 \mathrm{~nm}$ to beyond $1600 \mathrm{~nm}$ - range in which the sum of pump photon energy and signal photon energy is always higher than the bandgap of silicon.

\section{Conclusions}

We demonstrated all-optical wavelength conversion in submicron size silicon wire waveguides using ultrafast nonlinear TPA process at $1 \mathrm{GHz}$ repetition rate. Our results showed that silicon waveguides have potential applications in high speed photonic signal processing. The proposed scheme has the advantage of extremely wide wavelength conversion range, since it can potentially operate over the whole wavelength range where silicon is transparent (1200nm to beyond $1600 \mathrm{~nm})$.

\section{Acknowledgements}

We are indebt to J. Inoue, T. Isu, T. Kawanishi, M. Nakao, T. Sakamoto, K. Sasagawa, and H. Sotobayashi, for the fruitful discussions.

\section{References}

1 Tsuchizawa T. et al, J. Sel. Top. Quant. Electron. 11 (2005), 232-240.

2 O. Boyraz et al, Optics Express, vol. 12, no. 21 (2004), pp. 5269-5273.

3 Raghunathan V. et al, IEICE Electronics express 11 (2004), 298-304.

4 Day I. et al, OFC 2003, 249.

5 Almeida V.R. et al, Opt. Lett. 29 (2004), 28672869

6 Dimitropoulos D.R. et al. Appl. Phys Lett 86 (2005) Article 071115.

7 Tsang HK et al, J. Appl. Phys. 70 (1991), 39923994.

8 Bogaerts W. et al, J. Of Lightwave Technol. 23 (2005), 401-402.

9 Rauscher C. et al, J. Appl. Phys. 81 (1997), 28182820. 